\title{
Front Matter: Volume 7353
}

, "Front Matter: Volume 7353," Proc. SPIE 7353, Metamaterials IV, 735301 (2 June 2009); doi: 10.1117/12.834612

SPIE. Event: SPIE Optics + Optoelectronics, 2009, Prague, Czech Republic 


\title{
PROCEEDINGS OF SPIE
}

\section{Metamaterials IV}

\author{
Vladimir Kuzmiak \\ Peter Markoš \\ Tomasz Szoplik \\ Editors
}

\section{0-22 April 2009 \\ Prague, Czech Republic}

Sponsored by

SPIE Europe

Cooperating Organizations

Institute of Physics, Academy of Sciences (Czech Republic)

Department of X-Ray Lasers, Institute of Physics (Czech Republic)

Czech and Slovak Society for Photonics (Czech Republic)

Photonics Society of Poland (Poland)

Published by

SPIE 
The papers included in this volume were part of the technical conference cited on the cover and title page. Papers were selected and subject to review by the editors and conference program committee. Some conference presentations may not be available for publication. The papers published in these proceedings reflect the work and thoughts of the authors and are published herein as submitted. The publisher is not responsible for the validity of the information or for any outcomes resulting from reliance thereon.

Please use the following format to cite material from this book:

Author(s), "Title of Paper," in Metamaterials IV, edited by Vladimir Kuzmiak, Peter Markoš, Tomasz Szoplik, Proceedings of SPIE Vol. 7353 (SPIE, Bellingham, WA, 2009) Article CID Number.

ISSN 0277-786X

ISBN 9780819476272

Published by

SPIE

P.O. Box 10, Bellingham, Washington 98227-0010 USA

Telephone +1 3606763290 (Pacific Time) · Fax +1 3606471445

SPIE.org

Copyright (C) 2009, Society of Photo-Optical Instrumentation Engineers

Copying of material in this book for internal or personal use, or for the internal or personal use of specific clients, beyond the fair use provisions granted by the U.S. Copyright Law is authorized by SPIE subject to payment of copying fees. The Transactional Reporting Service base fee for this volume is $\$ 18.00$ per article (or portion thereof), which should be paid directly to the Copyright Clearance Center (CCC), 222 Rosewood Drive, Danvers, MA 01923. Payment may also be made electronically through CCC Online at copyright.com. Other copying for republication, resale, advertising or promotion, or any form of systematic or multiple reproduction of any material in this book is prohibited except with permission in writing from the publisher. The CCC fee code is 0277-786X/09/\$18.00.

Printed in the United States of America.

Publication of record for individual papers is online in the SPIE Digital Library.

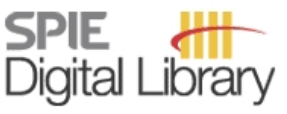

SPIEDigitalLibrary.org

Paper Numbering: Proceedings of SPIE follow an e-First publication model, with papers published first online and then in print and on CD-ROM. Papers are published as they are submitted and meet publication criteria. A unique, consistent, permanent citation identifier (CID) number is assigned to each article at the time of the first publication. Utilization of CIDs allows articles to be fully citable as soon they are published online, and connects the same identifier to all online, print, and electronic versions of the publication. SPIE uses a six-digit CID article numbering system in which:

- The first four digits correspond to the SPIE volume number.

- The last two digits indicate publication order within the volume using a Base 36 numbering system employing both numerals and letters. These two-number sets start with 00, 01, 02, 03, 04 , $05,06,07,08,09,0 A, 0 B \ldots$. OZ, followed by 10-1Z, 20-2Z, etc.

The CID number appears on each page of the manuscript. The complete citation is used on the first page, and an abbreviated version on subsequent pages. Numbers in the index correspond to the last two digits of the six-digit CID number. 


\section{Contents}

vii Conference Committee

ix Introduction

xi Photon physics: from wave mechanics to quantum electrodynamics (Plenary Paper) [7355-100]

O. Keller, Aalborg Univ. (Denmark)

\section{SESSION 1 METAMATERIALS I}

735302 Spatial dispersion in a wire mesh metamaterial (Invited Paper) [7353-01]

D. Felbacq, B. Guizal, Univ. Montpellier II, Groupe d'Etude des Semiconductors, CNRS

(France); G. Bouchitté, C. Bourel, Univ. du Sud-Toulon-Var, Lab. IMATH (France)

735303 Low-loss infrared metallo-dielectric metamaterials: theory and applications [7353-02]

B. Kanté, A. de Lustrac, J.-M. Lourtioz, IEF, Univ. Paris-Sud, CNRS (France)

735304 Negative and imaginary permittivity in 2D photonic macroporous silicon structures [7353-03] L. Karachevtseva, V. Ivanov, V. Onishchenko, O. Stronska, V. Lashkariov Institute of Semiconductor Physics (Ukraine)

735305 Negative effective permeability of multilayers of ordered arrays of metal-dielectric nanosandwiches [7353-04]

C. Tserkezis, N. Stefanou, G. Gantzounis, Univ. of Athens (Greece); N. Papanikolaou, Institute of Microelectronics (Greece)

\section{SESSION 2 METAMATERIALS II}

735308 Metamaterial absorber with wide angular and frequency bandwidth [7353-07]

A. Toscano, L. Vegni, Univ. degli Studi di Roma Tre (Italy)

735309 Infrared metamaterials and plasmons engineering [7353-08]

B. Kanté, A. de Lustrac, J.-M. Lourtioz, IEF, Univ. Paris-Sud, CNRS (France)

7353 OA Propagation and tunneling of electromagnetic waves through uniaxial metamaterials at arbitrary orientations of the optical axis [7353-09]

E. Starodubtsev, Gomel State Technical Univ. (Belarus)

7353 OB Optics of metamaterials based on channeled mirror structures [7353-10]

E. Ya. Glushko, Institute of Semiconductor Physics (Ukraine) 
7353 OC Analysis of metallic nanostructures via a discontinuous-Galerkin time-domain approach (Invited Paper) [7353-11]

K. Stannigel, M. König, J. Niegemann, K. Busch, Univ. Karlsruhe (Germany)

7353 OD Analytical modelling of linear and nonlinear properties of metamaterials based on multipole expansion [7353-12]

J. Petschulat, A. Chipouline, E. Pshenay-Severin, Friedrich-Schiller-Univ. Jena (Germany); A. Tünnermann, Friedrich-Schiller-Univ. Jena (Germany) and Fraunhofer Institute for Applied Optics and Precision Engineering (Germany); T. Pertsch, C. Menzel, C. Rockstuhl, T. Paul, F. Lederer, Friedrich-Schiller-Univ. Jena (Germany)

7353 OE Monte Carlo analysis of local distribution of negative refractive index in nanosphere-doped liquid crystal metamaterial [7353-13]

M. Jarema, W. Walasik, G. Pawlik, R. Orlik, A. C. Mitus, Wroclaw Univ. of Technology (Poland)

7353 OF Magneto-optical response enhancement in 1D and 2D magnetoplasmonic crystals [7353-14]

A. A. Grunin, A. G. Zhdanov, B. B. Tsema, A. A. Ezhov, T. V. Dolgova, E. A. Ganshina, Lomonosov Moscow State Univ. (Russian Federation); M. H. Hong, National Univ. of Singapore (Singapore); A. A. Fedyanin, Lomonosov Moscow State Univ. (Russian Federation)

7353 OG An all-purpose three-dimensional finite element model for crossed-gratings [7353-15] G. Demésy, Univ. Aix-Marseille III (France) and STMicroelectronics (France); F. Zolla, A. Nicolet, M. Commandré, Univ. Aix-Marseille III (France)

$7353 \mathrm{OH} \quad$ A genetic algorithm based procedure to retrieve effective parameters of planar metamaterial samples [7353-16]

S. Tricarico, F. Bilotti, L. Vegni, Univ. degli Studi di Roma Tre (Italy)

7353 ol Dielectric-metal-dielectric nanotip for SNOM [7353-48]

T. J. Antosiewicz, P. Wróbel, T. Szoplik, Univ. of Warsaw (Poland)

\section{SESSION $4 \quad$ PLASMONICS I}

7353 OK Propagation of surface plasmons through planar interface [7353-19]

T. Váry, P. Markoš, Slovak Univ. of Technology (Slovakia)

735300 The negative refractive index metamaterials as the aggregate of spherical particles or porous disposed in the different ambient medium [7353-23]

M. Tagviashvili, V. Berezhiani, E. Andronikashvili Institute of Physics (Georgia) 
7353 OS Transmission enhancement of light through a metallic nano-slit with periodic metallic nanostrips [7353-27]

Y. Cui, Zhejiang Univ. (China) and Joint Research Ctr. of Photonics of the Royal Institute of Technology (Sweden) and Zhejiang Univ. (China); Y. Jin, Joint Research Ctr. of Photonics of the Royal Institute of Technology (Sweden) and Zhejiang Univ. (China); Y. Okuno, Kumamoto Univ. (Japan); S. He, Joint Research Ctr. of Photonics of the Royal Institute of Technology (Sweden) and Zhejiang Univ. (China)

7353 OT Plasmon-induced wavelength-dependent polarization switching in optical metamaterials [7353-28]

M. R. Shcherbakov, P. P. Vabishchevich, T. V. Dolgova, Lomonosov Moscow State Univ. (Russian Federation); A. A. Zaitsev, A. S. Sigov, Moscow Institute for Radioengineering, Electronics and Automation (Russian Federation); A. A. Fedyanin, Lomonosov Moscow State Univ. (Russian Federation)

\section{SESSION 6 SUBWAVELENGTH IMAGING}

7353 OW Metal-dielectric superlens with ultra-flat phase of the modulation transfer function [7353-31] T. Stefaniuk, R. Kotyński, T. Szoplik, Univ. of Warsaw (Poland)

7353 0X Focusing of radially polarized light with corrugated silver nanolayer [7353-32]

P. Wrobel, J. Pniewski, T. J. Antosiewicz, T. Szoplik, Univ. of Warsaw (Poland)

7353 OY Subwavelength focusing using radiationless inteference at optical wavelengths [7353-33]

R. Gordon, Univ. of Victoria (Canada)

\section{SESSION 7 METAMATERIALS FABRICATION TECHNOLOGIES}

735310 Proximity-effect induced limitations on the density of electron-beam patterned planar photonic nanostructures [7353-35]

R. Wüest, ETH Zürich (Switzerland) and ABB Ltd. (Switzerland)

735311 Optical spectroscopy of terbium-scandium-aluminium garnet and terbium-scandium perovskite [7353-36]

K. Postava, L. Halagačka, D. Hrabovský, O. Životský, J. Pištora, Technical Univ. of Ostrava (Czech Republic); D. A. Pawlak, S. Turczynski, K. Kolodziejak, Institute of Electronic Materials Technology (Poland)

735312 Design of miniaturized printed monopole antennas through phase-compensation [7353-37]

L. Scorrano, F. Bilotti, L. Vegni, Univ. degli Studi di Roma Tre (Italy)

\section{SESSION 8 DEVICE APPLICATION OF METAMATERIALS}

735315 A quasi-quantitative demonstration of multi-mode refractive index sensors based on standing-wave plasmonic resonances in split ring resonators [7353-40]

Y.-T. Chang, T.-J. Yen, National Tsing Hua Univ. (Taiwan) 
POSTER SESSION

735319 Imaging in the visible wavelength range through anisotropic layered flat lens operating in the canalization regime [7353-44]

A. Pastuszczak, R. Kotyński, Univ. of Warsaw (Poland)

7353 1A Zero-average index band-gap edges in $\boldsymbol{m}$-bonacci metamaterial multilayers [7353-45]

J. A. Monsoriu, Univ. Politécnica de Valencia (Spain); R. A. Depine, M. L. Martínez-Ricci, Univ. de Buenos Aires (Argentina); P. Andrés, E. Silvestre, Univ. de Valencia (Spain)

7353 1C Complex Fourier factorization method applied in modeling optical metamaterials based on 2D periodic nanostructures [7353-47]

R. Antos, M. Veis, S. Visnovsky, Charles Univ. (Czech Republic)

Author Index 


\title{
Conference Committee
}

\author{
Symposium Chairs
}

Pavel Tomanek, Brno University of Technology (Czech Republic)

Alan G. Michette, King's College London (United Kingdom)

Bahaa E. A. Saleh, Boston University (United States)

Symposium Honorary Chair

Jan Perina, Sr., Palacký University (Czech Republic)

Conference Chairs

Vladimir Kuzmiak, Institute of Photonics and Electronics (Czech Republic)

Peter Markoš, Slovak University of Technology (Slovakia)

Tomasz Szoplik, University of Warsaw (Poland)

Program Committee

Nader Engheta, University of Pennsylvania (United States)

Didier Felbacq, Université Montpellier II (France)

Fransisco Javier Garcia de Abajo, Consejo Superior de Investigaciones Científicas (Spain)

Nigel P. Johnson, University of Glasgow (United Kingdom)

Maria Kafesaki, Institute of Electronic Structure and Laser of the Foundation for Research and Technology-Hellas (Greece)

Yuri S. Kivshar, The Australian National University (Australia)

Didier Lippens, Institut d'Electronique de Microélectronique et de Nanotechnologie (France)

Ekmel Özbay, Bilkent Üniversitesi (Turkey)

Dorota A. Pawlak, Instytut Technologii Materialów Elektronicznych (Poland)

Concita Sibilia, Università degli Studi di Roma, La Sapienza (Italy)

Costas M. Soukoulis, lowa State University (United States)

Sergei Tretyakov, Helsinki University of Technology (Finland)

Martin Wegener, Universität Karlsruhe (Germany)

Nikolay I. Zheludev, University of Southampton (United Kingdom)

Richard W. Ziolkowski, The University of Arizona (United States) 
Session Chairs

1 Metamaterials I

Kurt Busch, Universität Karlsruhe (Germany)

2 Metamaterials II

Peter Markoš, Slovak University of Technology (Slovakia)

3 Modelling of Metamaterials

Vladimir Kuzmiak, Institute of Photonics and Electronics (Czech Republic)

$4 \quad$ Plasmonics I

Stavroula Foteinopoulou, Foundation for Research and Technology-Hellas (Greece)

5 Plasmonics II

Concita Sibilia, Università degli Studi di Roma, La Sapienza (Italy)

6 Subwavelength Imaging

Vladimir Kuzmiak, Institute of Photonics and Electronics

(Czech Republic)

$7 \quad$ Metamaterials Fabrication Technologies

Constantin R. Simovski, University ITMO (Russian Federation)

8 Device Application of Metamaterials

Tomasz Szoplik, University of Warsaw (Poland) 


\section{Introduction}

Metamaterials have become an influential concept which has a profound impact on physics, optics, and engineering communities. Unique electromagnetic features of metamaterials that have been demonstrated over the past two decades have created prospects of advancement in new applications such as cloaking, superlensing, and hyperlensing. When the metamaterial features are not limited to negative refraction only, similarity of properties of metamaterials, photonic crystals, plasmonic, and metal-dielectric multilayer structures lead to entirely new limits of knowledge and unexpected applications.

This fourth conference (the second one held in Prague) in a series of SPIE conferences on metamaterials brought together the two scientific communities of metamaterials and plasmonics. It provided an overview of recent activities in the field of complex metamaterial structures, their coupling properties, hybridization of both the electric and magnetic resonances, and latest achievements in tunable and nonlinear metamaterials. Both device applications and progress in modeling metamaterials were presented. New concepts of plasmonic devices for light transport in the subwavelength scale and their use in nano-optics systems were discussed in several interesting presentations.

This conference was one of 10 conferences held at the SPIE Europe Congress on Optics and Optoelectronics organized in Prague by the Institute of Physics, Academy of Science of the Czech Republic and SPIE Europe. The Congress brought together leading scientists to an important Middle Europe forum addressing the most important developments in the field of photonics and optoelectronics.

As chairs of this meeting we would like to express our thanks to all those participants who contributed through their presentations and to the program committee members.

Vladimir Kuzmiak

Peter Markoš

Tomasz Szoplik 
Downloaded From: https://www.spiedigitallibrary.org/conference-proceedings-of-spie on 26 Apr 2023

Terms of Use: https://www.spiedigitallibrary.org/terms-of-use 


\title{
PHOTON PHYSICS: FROM WAVE MECHANICS TO QUANTUM ELECTRODYNAMICS
}

\author{
Ole Keller \\ Institute of Physics and Nanotechnology, Aalborg University, Denmark
}

\begin{abstract}
When rewritten in an appropriate manner, the microscopic Maxwell-Lorentz equations appear as a wavemechanical theory for photons, and their quantum physical interaction with matter. A natural extension leads from photon wave mechanics to quantum electrodynamics (QED). In its modern formulation photon wave mechanics has given us valuable new insight in subjects such as spatial photon localization, near-field photon dynamics, transverse photon mass, photon eikonal theory, photon tunneling, and rim-zone electrodynamics. The present review is based on my plenary lecture at the SPIE-Europe 2009 Optics and Optoelectronics International Symposium in Prague.
\end{abstract}

\section{INTRODUCTION}

The birth of quantum field radiation theory in the years 1925-30, did not cause physicists to give up the idea of a particle concept of light. Beautiful wave mechanical theories for the photon were thus established by Landau and Peierls in $1930,{ }^{1}$ and by Oppenheimer ${ }^{2}$ the year after. For a brief historical review of photon wave mechanics the reader may consult reference three. ${ }^{3}$ Over the years physicists interest in photon wave mechanics, and its extension to the field-quantized level, has waxed and waned, but never fallen to rest. ${ }^{4}$ In the present review, I look at this almost century old field in a modern perspective, and point out some of the modern perspectives for low-energy physical optics.

It has been known for almost two centuries that the eikonal equation in geometrical optics is form-identical to the Hamilton-Jacobi equation for Hamilton's characteristic function in Newtonian mechanics. ${ }^{5,6}$ This fact led almost exactly a century later De Broglie and Schrödinger to the wave mechanics of massive particles. ${ }^{3,5,6}$ In this perspective, it is perhaps not surprising that classical electrodynamics, based on the microscopic MaxwellLorentz equations, may be looked upon as the wave-mechanical theory of the photon. Starting from the KleinGordon equation, the quantum-mechanical eikonal equation for a relativistic spinless particle subjected to an electromagnetic field is first established.

In free space various constructions (objects) can claim to represent a photon wave function. Here, we discuss two of the most attractive suggestions: the photon energy wave function, ${ }^{2,4}$ and a wave function based on the transverse vector potential. ${ }^{7}$ For the potential approach, we set up the photon Schrödinger equation in both momentum and configuration space. Afterwards, we discuss the transverse photon mass concept in diamagnetic field-matter interaction, and the photon eikonal theory.

Modern photon wave mechanics appears to be particularly important in the quantum theory of near-field electrodynamics. ${ }^{8,9}$ In the rim zone of matter virtual photons play a significant role, but only in light-matter interactions. A (new) virtual photon type called a near-field photon is introduced and its wave mechanics analyzed. The near-field photon is accompanied by a gauge photon, and the two photons place in QED is discussed. I end the review by a brief discussion of the position operator problem for massive photons (plasmaritons), and emphasize the importance of the rim zone in treatments of the spatial localization problem for photons emitted from microscopic (or mesoscopic) sources. ${ }^{10,11}$

Photon Counting Applications, Quantum Optics, and Quantum Information Transfer and Processing II, edited by Ivan Prochazka, Roman Sobolewski, Miloslav Dusek, Proc. of SPIE Vol. 7355, 735502 (C) 2009 SPIE · CCC code: 0277-786X/09/\$18 - doi: 10.1117/12.833543 


\section{GEOMETRICAL OPTICS AND NEWTONIAN MECHANICS IN THE HAMILTON-JACOBI FORMULATION}

It has been known for almost two centuries that the dynamics of a system of interacting classical (Newtonian) particles can be described neatly on the basis of Hamilton's equations

$$
\begin{aligned}
\dot{q}_{i} & =\frac{\partial}{\partial p_{i}} H(\{q\},\{p\}, t) \\
\dot{p}_{i} & =-\frac{\partial}{\partial q_{i}} H(\{q\},\{p\}, t)
\end{aligned}
$$

where $q_{i}$ is the generalized coordinate number $i$, and $p_{i}$ its associated canonical (conjugate) momentum. The dots stand for differentiation with respect to time. As indicated, the Hamilton function $H$ depends in general on the full set of coordinates and momenta, and on time. By a particular canonical transformation, which generating function involves Hamilton's principal function $S(\{q\},\{P\}, t)$, the new Hamiltonian can be required to vanish identically. This ensures that the new generalized coordinates and momenta $(\{P\})$ become time-independent, and can be taken as the initial values of the old phase space variables. Hamilton's principal function now must satisfy the partial differential equation (called the Hamilton-Jacobi equation)

$$
H\left(\{q\},\left\{\frac{\partial S}{\partial q}\right\}, t\right)+\frac{\partial S}{\partial t}=0,
$$

and the (old) generalized momenta are identified as

$$
p_{i}=\frac{\partial}{\partial q_{i}} S(\{q\}, t),
$$

leaving out the reference to $\{P\}$ from the notation. In the special but important case where $H$ does not depend explicitly on time, a trial solution of the form

$$
S(\{q\}, t)=W(\{q\})-E t
$$

leads to the Hamilton-Jacobi equation

$$
H\left(\{q\},\left\{\frac{\partial W}{\partial q}\right\}\right)=E
$$

for Hamilton's characteristic function, $W(\{q\})$. Usually, the constant E represents the (conserved) energy of the system. For a single particle in a time-independent potential, $V(\mathbf{r})$, the equation for Hamilton's characteristic function takes the following form:

$$
\nabla W(\mathbf{r}) \cdot \nabla W(\mathbf{r})=2 m(E-V(\mathbf{r})),
$$

where $m$ is the particle's mass, and $\mathbf{r}$ its position vector. With the identification in Eq. (4), which here on vector form gives $\mathbf{p}=\nabla W$, Eq. (7) expresses the fact that the particle's total energy is the sum of the kinetic and potential energies.

Geometrical optics deals with the phenomena for which the wavelength of light can be neglected. In this short-wavelength limit $(\lambda \rightarrow 0)$ the optical laws may be formulated in the language of geometry, and the basic quantity is the so-called eikonal $S(\mathbf{r})$, which enters the various (monochromatic) electromagnetic field vectors $(\mathbf{V})$ via the ansatz $\mathbf{V}(\mathbf{r})=\mathbf{V}_{0} \exp \left(i q_{0} S(\mathbf{r})\right), q_{0}=\omega / c_{0}$ being the vacuum wavenumber of light at the angular frequency $\omega$. The fundamental equations of geometrical optics is the eikonal equation ${ }^{6}$

$$
\nabla S(\mathbf{r}) \cdot \nabla S(\mathbf{r})=n^{2}(\mathbf{r}),
$$

where $n(\mathbf{r})$ is the refractive index, which in the lowest-order approximation is taken as a real quantity. As first noticed by Hamilton in 1827 there is a striking form-identity between the characteristic function [Eq. (7)] and the eikonal equation of geometrical optics [Eq. (8)]. Classical electrodynamics based in the macroscopic Maxwell equations (or in a somewhat broader perspective the microscopic Maxwell-Lorentz equations) is the covering 
theory of geometrical optics, and this fact led almost exactly one hundred years later De Broglie and Schrödinger to the covering theory of Newtonian mechanics, i.e., quantum mechanics, as might be well-known to the reader.

For what follows it is valuable briefly to consider the quantum mechanical eikonal theory for a relativistic spinless particle of rest mass $m$ and charge $q$. Placed in an electromagnetic field described by the contravariant four-potential $\left\{A^{\mu}\right\}=\left(A^{0}=\Phi / c_{0}, \mathbf{A}\right)$ the classical relativistic Hamiltonian is

$$
H=c_{0}\left[\left(m c_{0}\right)^{2}+(\mathbf{p}-q \mathbf{A})^{2}\right]^{1 / 2}+q \Phi .
$$

The Hamiltonian in Eq. (9) has no explicit time dependence, and with $\mathbf{p}=\nabla S$, the equation for Hamilton's principal function [Eq. (3)] can be written down. All solutions to this equation will also be solutions to the squared Hamilton-Jacobi equation, viz.,

$$
c_{0}^{2}(\nabla S-q \mathbf{A})^{2}+\left(m c_{0}^{2}\right)^{2}=\left(\frac{\partial S}{\partial t}+q \Phi\right)^{2} .
$$

The quantum mechanical eikonal equation for a relativistic spinless particle subjected to an electromagnetic field is obtained starting from the Klein-Gordon equation

$$
c_{0}^{2}\left[\left(\frac{\hbar}{i} \nabla-q \mathbf{A}\right)^{2}+\left(m c_{0}\right)^{2}\right] \Psi(\mathbf{r}, t)=\left(\frac{\hbar}{i} \frac{\partial}{\partial t}+q \Phi\right)^{2} \Psi(\mathbf{r}, t) .
$$

By inserting the eikonal ansatz

$$
\Psi(\mathbf{r}, t)=\Psi_{0} \exp \left(\frac{i}{\hbar} S(\mathbf{r}, t)\right)
$$

for the wavefunction, the quantum mechanical Hamilton-Jacobi equation takes the form

$$
(\nabla S-q \mathbf{A})^{2}+\left(m c_{0}\right)^{2}-\frac{1}{c_{0}^{2}}\left(\frac{\partial S}{\partial t}+q \Phi\right)^{2}=i \hbar\left(\nabla^{2}-\frac{1}{c_{0}^{2}} \frac{\partial^{2}}{\partial t^{2}}\right) S
$$

in the Lorenz gauge. In the classical limit $(\hbar \rightarrow 0)$, Eq. (13) is reduced to Eq. (10). In covariant notation, one has in general

$$
\left(\partial_{\mu} S-q A_{\mu}\right)\left(\partial^{\mu} S-q A^{\mu}\right)+\left(m c_{0}\right)^{2}=i \hbar \partial_{\mu}\left(\partial^{\mu} S-q A^{\mu}\right),
$$

and with the Lorenz gauge condition, given by

$$
\partial_{\mu} A^{\mu}=0
$$

Eq. (14) is reduced to the form given in Eq. (13). The relativistic covariance of the eikonal equation is manifest when the equation is written in the form given in Eq. (14).

\section{PHOTON WAVE MECHANICS}

The analysis in section 2 suggests that the microscopic Maxwell-Lorentz equations somehow may be considered as a wave-mechanical theory of the photon. This possibility was originally investigated by Landau and Peierls ${ }^{1}$ and Oppenheimer, ${ }^{2}$ and over the years physicist's interest in photon wave mechanics has waxed and waned, but never fallen to rest. In free space, various "constructions" (objects) can rightly claim that they represent the wave function of the photon. What is important physically is that the observable predictions related to the photon-matter interaction will be identical for the various choices.

In the energy wave-function formalism two photon wave functions, $\mathbf{f}_{ \pm}^{(+)}$, are defined as follows: ${ }^{4}$

$$
\mathbf{f}_{ \pm}^{(+)}(\mathbf{r}, t)=\left(\frac{\epsilon_{0}}{2}\right)^{1 / 2}\left[\mathbf{e}_{T}^{(+)}(\mathbf{r}, t) \pm i c_{0} \mathbf{b}^{(+)}(\mathbf{r}, t)\right] .
$$

The superscript $(+)$ indicates that the wave function is build from positive-frequency components of the electromagnetic field. In the language of coherence theory the $\mathbf{f}_{ \pm}^{(+)}$'s are analytical signals. The wave function of 
the antiphoton is constructed from the negative-frequency part of the field spectrum. Physically, the photon and antiphoton are identical. This can be inferred from the Maxwell equations. Small letters $\mathbf{e}_{T}$ and $\mathbf{b}$ have been assigned to the (analytical) electric and magnetic fields of the photon to indicate that the wave function has been properly normalized. To normalize the wave function of the in general non-monochromatic photon it is necessary to adjust the amplitude of the current-density source distribution correctly. ${ }^{8}$ To emphasize that the electrical field in free space is a transverse vector field, a subscript $T$ has been added to $\mathbf{e}^{(+)}$. As we shall understand very soon, $\mathbf{f}_{+}^{(+)}$and $\mathbf{f}_{-}^{(+)}$are wave functions composed of positive- and negative-helicity components. The photon helicity is a Lorentz-invariant property.

From the free-space Maxwell equations one can establish the following quantum mechanical wave equations for the two helicity species:

$$
i \hbar \frac{\partial}{\partial t} \mathbf{f}_{ \pm}^{(+)}(\mathbf{r}, t)= \pm c_{0}\left(\frac{\mathbf{s}}{\hbar} \cdot \frac{\hbar}{i} \nabla\right) \mathbf{f}_{ \pm}^{(+)}(\mathbf{r}, t) .
$$

In Eq. (17) we have written the wave equation in Hamiltonian form. The vector $\mathbf{s}$ is the Cartesian spin-one operator of the photon. In a $3 \times 3$ matrix representation the Cartesian components of $\mathbf{s}$ are given by

$$
\left(s_{k}\right)_{i j}=\frac{\hbar}{i} \epsilon_{i j k}
$$

where $\epsilon_{i j k}$ is the Levi-Civita tensor. Since $\hat{\mathbf{p}}=(\hbar / i) \nabla$ is the photon momentum operator in configuration space, the scalar product $\mathbf{s} \cdot \hat{\mathbf{p}}$ confirms that the wave functions $\mathbf{f}_{ \pm}^{(+)}(\mathbf{r}, t)$ describe photons composed of positiveand negative helicity components. Since Plancks constant $(h)$ does not appear in the classical Maxwell-Lorentz equations, $\hbar$ can of course be eliminated from Eq. (17), as the reader readily may realize. The name photon energy wave function relates to the fact that the quantum mechanical mean value of the photon energy-momentum four vector operator $\left\{p^{\mu}\right\}=\left(\hbar \omega / c_{0}, \hbar \mathbf{q}\right)$ is given by

$$
\left\{P^{\mu}\right\}=\frac{1}{\hbar c_{0}} \sum_{s=+,-} \int_{-\infty}^{\infty}\left\{p^{\mu}\right\}\left|f_{s}^{(+)}(\mathbf{q} ; t)\right|^{2} \frac{d^{3} q}{(2 \pi)^{3} q},
$$

where the $f_{ \pm}^{(+)}(\mathbf{q} ; t)$ 's are the scalar photon wave functions in momentum space.

The vectorial and scalar wave functions are related by $\mathbf{f}_{ \pm}^{(+)}(\mathbf{q} ; t)=f_{ \pm}^{(+)}(\mathbf{q} ; t) \mathbf{e}_{ \pm}(\mathbf{q} / q)$, where $\mathbf{e}_{ \pm}$are the helicity unit vectors of the + and - species. Relativistically, $\bar{d}^{3} q / q$ is the invariant volume element on the light cone.

It is often convenient to use spinor notation, and thus combine the two wave functions in Eq. (16) into a single one, viz.,

$$
\boldsymbol{\Phi}(\mathbf{r}, t)=\left(\begin{array}{c}
\mathbf{f}_{+}^{(+)}(\mathbf{r}, t) \\
\mathbf{f}_{-}^{(+)}(\mathbf{r}, t)
\end{array}\right) .
$$

The photon wave function in Eq. (20) is a six-component object.

In the present context it is useful to base photon wave mechanics on the electromagnetic potentials. In free space only a transverse vector potential exists, and this potential is gauge invariant. The analytical part of the transverse vector potential $\mathbf{a}_{T}^{(+)}(\mathbf{r}, t)$ satisfies the wave equation

$$
\square \mathbf{a}_{T}^{(+)}(\mathbf{r}, t)=\mathbf{0},
$$

where $\square=\nabla^{2}-c_{0}^{-2} \partial^{2} / \partial t^{2}$ is the d'Lambertian operator. Formally, one may factorize the $\square$-operator as follows:

$$
\square=\left(\frac{i}{c_{0}} \frac{\partial}{\partial t}+\sqrt{-\nabla^{2}}\right)\left(\frac{i}{c_{0}} \frac{\partial}{\partial t}-\sqrt{-\nabla^{2}}\right),
$$

and from this it appears that all solutions to

$$
i \hbar \frac{\partial}{\partial t} \mathbf{a}_{T}^{(+)}(\mathbf{r}, t)=\hbar c_{0} \sqrt{-\nabla^{2}} \mathbf{a}_{T}^{(+)}(\mathbf{r}, t)
$$


are also solutions to Eq. (21). One may consider $\mathbf{a}_{T}^{(+)}(\mathbf{r}, t)$ [small letter for normalization] as the vectorial wave function for the photon in free space, and Eq. (23) as its quantum mechanical wave equation, written in Hamiltonian form. The quantity $\sqrt{-\nabla^{2}}$ is a spatially nonlocal operator which action in Fourier (wave-vector) space is given via

$$
\sqrt{-\nabla^{2}} \mathbf{a}_{T}^{(+)}(\mathbf{r}, t)=\int_{-\infty}^{\infty} q \mathbf{a}_{T}^{(+)}(\mathbf{q} ; t) e^{i \mathbf{q} \cdot \mathbf{r}} \frac{d^{3} q}{(2 \pi)^{3}}
$$

The operator $i \sqrt{-\nabla^{2}}$ was originally introduced by Landau and Peierls in 1930 in connection with their attempt to establish a wave equation for the light quantum (photon). In q-space, Eq. (23) takes the form

$$
i \hbar \frac{\partial}{\partial t} \mathbf{a}_{T}^{(+)}(\mathbf{q} ; t)=c_{0} \hbar q \mathbf{a}_{T}^{(+)}(\mathbf{q} ; t)
$$

and this equation may be considered as the vectorial wave equation for a transverse photon with momentum $\mathbf{p}=\hbar \mathbf{q}$ and energy $H=p c_{0}$. Two transverse scalar photon wave functions can be introduced in the (q; $\left.t\right)$-domain by expanding $\mathbf{a}_{T}^{(+)}(\mathbf{q} ; t)$ after two orthogonal (possibly complex) unit vectors (helicity unit vectors, e.g) located in a plane perpendicular to $\mathbf{q}$.

\section{TRANSVERSE PHOTON MASS. PHOTON EIKONAL THEORY}

Let us now consider the photon propagation in a solid-state plasma (jellium), and let us limit ourselves to high frequencies and linear photon-matter interactions. At high frequencies the particle properties of the photon dominates over its wave properties, and the field-matter coupling is diamagnetic. In the space-frequency domain the analytical part of the transverse vectorial potential hence satisfies the integro-differential equation ${ }^{12}$

$$
\left(\nabla^{2}+q_{0}^{2}\right) \mathbf{a}_{T}^{(+)}(\mathbf{r} ; \omega)=\frac{\mu_{0} e^{2}}{m} \int_{-\infty}^{\infty} \overleftrightarrow{\delta}_{T}\left(\mathbf{r}-\mathbf{r}^{\prime}\right) \cdot\left[N\left(\mathbf{r}^{\prime}\right) \mathbf{a}_{T}^{(+)}\left(\mathbf{r}^{\prime} ; \omega\right)\right] d^{3} r^{\prime}
$$

where $N(\mathbf{r})$ is the many-body electron density, $\overleftrightarrow{\delta}_{T}\left(\mathbf{r}-\mathbf{r}^{\prime}\right)$ is the transverse delta function, $q_{0}=\omega / c_{0}$ is the vacuum wave number of light, and $e$ and $m$ the electron charge and mass. In the present context there is a particularly interesting special case of Eq. (26). Thus, if the electron density variations in space are negligible $\left[N(\mathbf{r}) \cong N_{0}\right]$ it can be shown that $\mathbf{a}_{T}^{(+)}(\mathbf{r}, t)$ satisfies the equation

$$
i \hbar \frac{\partial}{\partial t} \mathbf{a}_{T}^{(+)}(\mathbf{r}, t)=c_{0} \hbar \sqrt{Q_{c}^{2}-\nabla^{2}} \mathbf{a}_{T}^{(+)}(\mathbf{r}, t)
$$

where the operator $\left(Q_{c}^{2}-\nabla^{2}\right)^{1 / 2}$ acts as follows in Fourier space:

$$
\sqrt{Q_{c}^{2}-\nabla^{2}} \mathbf{a}_{T}^{(+)}(\mathbf{r}, t)=\int_{-\infty}^{\infty} \sqrt{Q_{c}^{2}+q^{2}} \mathbf{a}_{T}^{(+)}(\mathbf{q} ; t) e^{i \mathbf{q} \cdot \mathbf{r}} \frac{d^{3} q}{(2 \pi)^{3}} .
$$

The quantity

$$
Q_{c}=\frac{1}{c_{0}}\left(\frac{N_{0} e^{2}}{m \epsilon_{0}}\right)^{1 / 2}
$$

is the electron system's plasma wave number. One may consider Eq. (27) as the quantum mechanical wave equation for the transverse photon (plasmariton). In the wave-vector-time domain Eq. (27) takes the form

$$
i \hbar \frac{\partial}{\partial t} \mathbf{a}_{T}^{(+)}(\mathbf{q} ; t)=c_{0} \hbar\left(q^{2}+Q_{c}^{2}\right)^{1 / 2} \mathbf{a}_{T}^{(+)}(\mathbf{q} ; t)
$$

and from this one may show that the energy-momentum relation for the plasmariton takes the relativistic form

$$
E=+\left[\left(p c_{0}\right)^{2}+\left(M c_{0}^{2}\right)^{2}\right]^{1 / 2}
$$


In a sense one may now claim that the transverse photon has acquired a finite mass

$$
M=\frac{\hbar Q_{c}}{c_{0}}
$$

in its high-frequency interaction with the jellium. The quantity $Q_{c}=M c_{0} / \hbar$ is the Compton wave number of the massive transverse photon.

The wave equation for the plasmariton is form-identical to the Klein-Gordon equation, and the eikonal ansatz $\mathbf{a}_{T}^{(+)}(\mathbf{r}, t)=\mathbf{a}_{T, 0}^{(+)} \exp (i S(\mathbf{r}, t) / \hbar)$ therefore leads to the following eikonal equation for the "free" massive photon:

$$
\left(\partial_{\mu} S\right)\left(\partial^{\mu} S\right)+\left(M c_{0}\right)^{2}=i \hbar \partial_{\mu} \partial^{\mu} S .
$$

If the spatial variations in $N(\mathbf{r})$ is taken into account the eikonal problem becomes more complicated.

\section{THE NEAR-FIELD PHOTON CONCEPT}

In near-field electrodynamics one must include two other types of photons, viz., longitudinal and scalar photons. These so-called virtual photons, which do not exist as free particles, we hence characterize by the analytical vector potentials $a_{L}^{(+)}(\mathbf{q} ; t)\left[\mathbf{a}_{L}^{(+)}=a_{L}^{(+)} \mathbf{q} / q\right]$ and $a_{0}^{(+)}(\mathbf{q} ; t)$. Instead of using the longitudinal and scalar photon variables it is convenient in near-field optics to introduce two new photon types via the unitary transformation ${ }^{9}$

$$
\left(\begin{array}{c}
a_{N F}^{(+)}(\mathbf{q} ; t) \\
a_{G}^{(+)}(\mathbf{q} ; t)
\end{array}\right)=\frac{1}{\sqrt{2}}\left(\begin{array}{cc}
i & -i \\
1 & 1
\end{array}\right)\left(\begin{array}{c}
a_{L}^{(+)}(\mathbf{q} ; t) \\
a_{0}^{(+)}(\mathbf{q} ; t)
\end{array}\right) .
$$

The quantities $a_{N F}^{(+)}(\mathbf{q} ; t)$ and $a_{G}^{(+)}(\mathbf{q} ; t)$ are the quantum mechanical wave functions of the near-field (NF) and gauge $(\mathrm{G})$ photons. The theory for $a_{L}^{(+)}$and $a_{0}^{(+)}$, or equivalently $a_{N F}^{(+)}$and $a_{G}^{(+)}$, always is formulated in the Lorenz gauge. The primary quantity in near-field electrodynamics is the analytical longitudinal electric field $\mathbf{E}_{L}^{(+)}(\mathbf{q} ; t)=E_{L}^{(+)}(\mathbf{q} ; t) \mathbf{q} / q$. It may be shown that the Lorenz gauge condition implies that $E_{L}^{(+)}(\mathbf{q} ; t)$ can be expressed solely in terms of either $a_{N F}^{(+)}(\mathbf{q} ; t)$ or $a_{G}^{(+)}(\mathbf{q} ; t)$, i.e.,

$$
E_{L}^{(+)}(\mathbf{q} ; t)=\sqrt{2}\left(c_{0} q+i \frac{\partial}{\partial t}\right) a_{N F}^{(+)}(\mathbf{q} ; t)=-i \sqrt{2}\left(c_{0} q-i \frac{\partial}{\partial t}\right) a_{G}^{(+)}(\mathbf{q} ; t) .
$$

In free space the near-field and gauge photon wave functions satisfy the Hamiltonian wave equations

$$
\begin{aligned}
& i \hbar \frac{\partial}{\partial t} a_{N F}^{(+)}(\mathbf{q} ; t)=c_{0} \hbar q a_{N F}^{(+)}(\mathbf{q} ; t), \\
& i \hbar \frac{\partial}{\partial t} a_{G}^{(+)}(\mathbf{q} ; t)=c_{0} \hbar q a_{G}^{(+)}(\mathbf{q} ; t),
\end{aligned}
$$

and since $E_{L}(\mathbf{q} ; t)=0$ in free space, Eqs. (35) and (36) imply that $a_{N F}^{(+)}(\mathbf{q} ; t)=0$. One cannot conclude from these equations that the wave function of the gauge photon vanishes in free space. A gauge transformation to a new (NEW) gauge within the Lorenz gauge can make $a_{G}^{(+) N E W}(\mathbf{q} ; t)=0$. The name gauge photon originates in this property. In the presence of matter, the near-field and gauge photon variables satisfy the dynamical equations

$$
\begin{aligned}
& \left(c_{0} q+i \frac{\partial}{\partial t}\right) a_{N F}^{(+)}(\mathbf{q} ; t)=\frac{1}{i \sqrt{2} \epsilon_{0}} \frac{1}{q c_{0}} J_{0}^{(+)}(\mathbf{q} ; t), \\
& \left(c_{0} q-i \frac{\partial}{\partial t}\right) a_{G}^{(+)}(\mathbf{q} ; t)=\frac{1}{\sqrt{2} \epsilon_{0}} \frac{1}{q c_{0}} J_{0}^{(+)}(\mathbf{q} ; t),
\end{aligned}
$$

where $J_{0}^{(+)}(\mathbf{q} ; t)=c_{0} \rho(\mathbf{q} ; t)$ is the $\mu=0$ component of the contravariant current density four-vector. 


\section{RIM ZONE ELECTRODYNAMICS}

The near-field photon concept connects to optical near-field interactions via Eq. (35) and (38). I shall not here undertake a systematic analysis of this connection. The longitudinal part of the electric field, $\mathbf{E}_{L}$, plays an important role in near-field optics, and this field relates to the near-field photon variable via the analytical relation

$$
\mathbf{E}_{L}^{(+)}(\mathbf{r}, t)=c_{0} \sqrt{2}\left(\frac{i}{c_{0}} \frac{\partial}{\partial t}+\sqrt{-\nabla^{2}}\right) \mathbf{a}_{N F}^{(+)}(\mathbf{r}, t),
$$

where $\mathbf{a}_{N F}^{(+)}(\mathbf{r}, t)$ is the spatial Fourier transform of the vectorial quantity $a_{N F}^{(+)}(\mathbf{q} ; t) \mathbf{q} / q$. As an immediate consequence of Eqs. (35) and (38) the reader may obtain the following relation between the longitudinal electric field and the longitudinal source current density $\left(\mathbf{J}_{L}\right)$ :

$$
\mathbf{J}_{L}(\mathbf{r} ; \omega)=i \epsilon_{0} \omega \mathbf{E}_{L}(\mathbf{r} ; \omega) .
$$

In the presence of a transverse current density source, $\mathbf{J}_{T}^{(+)}(\mathbf{r}, t)$, the transverse photon variable satisfies the inhomogeneous wave equation

$$
\square \mathbf{a}_{T}^{(+)}(\mathbf{r}, t)=-\mu_{0} \mathbf{J}_{T}^{(+)}(\mathbf{r}, t),
$$

and from this one can obtain the integral relation

$$
\mathbf{E}_{T}(\mathbf{r} ; \omega)=i \mu_{0} \omega \int_{-\infty}^{\infty} g\left(\left|\mathbf{r}-\mathbf{r}^{\prime}\right| ; \omega\right) \mathbf{J}_{T}\left(\mathbf{r}^{\prime} ; \omega\right) d^{3} r^{\prime}
$$

between the transverse parts of the electric field and source current density. The quantity $g(R ; \omega)=(4 \pi R)^{-1}$ $\times \exp \left(i q_{0} R\right)$ is the outgoing scalar propagator. The relations in Eqs. (41) and (43) form the standard starting point for classical near-field electrodynamics. Everywhere in space outside matter one has $\mathbf{J}_{L}=-\mathbf{J}_{T}$, since $\mathbf{J}=\mathbf{0}$. It appears from the equation

$$
\mathbf{J}_{L}(\mathbf{r} ; \omega)=\frac{1}{3} \mathbf{J}(\mathbf{r} ; \omega)+\frac{1}{4 \pi} P V \int_{-\infty}^{\infty} R^{-3}\left(\overleftrightarrow{\mathbf{U}}-3 \frac{\mathbf{R R}}{R^{2}}\right) \cdot \mathbf{J}\left(\mathbf{r}^{\prime} ; \omega\right) d^{3} r^{\prime}
$$

where $\mathbf{R}=\mathbf{r}-\mathbf{r}^{\prime}$, and PV stands for principal value that $\mathbf{J}_{L}$ is appreciably different from zero only in a narrow zone outside a matter-filled region. This zone I have called the rim zone, and in this zone the longitudinal electric field is nonvanishing, and satisfies

$$
\nabla \cdot \mathbf{E}_{L}(\mathbf{r} ; \omega)=\nabla \times \mathbf{E}_{L}(\mathbf{r} ; \omega)=0,
$$

the last equation per definition, of course. In the language of photon wave mechanics near-field optics describes how transverse photons via their interaction with near-field photons are generated and destroyed in space-time.

\section{NEAR-FIELD AND GAUGE PHOTONS IN QED}

The wave mechanical formalism can be extended to the quantum-electrodynamic level by the standard covariant quantization with an indefinite metric (Gupta-Bleuler formalism), ${ }^{13} \cdot{ }^{14}$ The Hamilton operator of the field becomes (in box quantization)

$$
\hat{H}=\sum_{\mathbf{q}, r} \hbar \omega_{q} \hat{a}_{r}^{\dagger}(\mathbf{q}) \hat{a}_{r}(\mathbf{q}),
$$

where $\omega_{q}=c_{0} q$. The r-summation in Eq. (46) is over the two transverse modes (T1, T2), and the longitudinal (L) and scalar $(0)$ modes. The annihilation $\left(\hat{a}_{r}(\mathbf{q})\right)$ and creation $\left(\hat{a}_{r}^{\dagger}(\mathbf{q})\right)$ operators of the various modes satisfy the commutation relations

$$
\left[\hat{a}_{r}(\mathbf{q}), \hat{a}_{s}^{\dagger}\left(\mathbf{q}^{\prime}\right)\right]=\delta_{r s} \delta_{\mathbf{q}, \mathbf{q}^{\prime}} .
$$

By extension of Eq. (34) to the operator level it is easy to show that

$$
\hat{a}_{L}^{\dagger} \hat{a}_{L}+\hat{a}_{0}^{\dagger} \hat{a}_{0}=\hat{a}_{N F}^{\dagger} \hat{a}_{N F}+\hat{a}_{G}^{\dagger} \hat{a}_{G},
$$


a relation which allows one to eliminate the longitudinal and scalar photon operators in the field Hamiltonian in favour of the near-field and gauge photon operators. The annihilation and creation operators of these virtual photon types satisfy the commutator relations

$$
\begin{gathered}
{\left[\hat{a}_{N F}(\mathbf{q}), \hat{a}_{N F}^{\dagger}\left(\mathbf{q}^{\prime}\right)\right]=\delta_{\mathbf{q}, \mathbf{q}^{\prime}},} \\
{\left[\hat{a}_{G}(\mathbf{q}), \hat{a}_{G}^{\dagger}\left(\mathbf{q}^{\prime}\right)\right]=\delta_{\mathbf{q}, \mathbf{q}^{\prime}},}
\end{gathered}
$$

and the NF-G commutators vanish. In free space the quantum Lorenz condition leads for all $\mathbf{q}$ to

$$
\left[\hat{a}_{L}(\mathbf{q})-\hat{a}_{0}(\mathbf{q})\right]|\psi\rangle=\hat{a}_{N F}(\mathbf{q})|\psi\rangle=0,
$$

so that no physical states $|\psi\rangle$ have near-field photons. The Lorenz condition puts no restrictions on the number of gauge photons in free space. Two different gauges within the Lorenz gauge correspond to two different excitations of G-modes, but as in the classical theory the gauge photon plays no physical role in free space.

\section{PLASMARITON POSITION OPERATOR. SPATIAL PHOTON LOCALIZATION}

Let us now ask the following question: to what extend can a point particle in the form of a transverse (massive) photon be localized in space? If a particle's position is to be measured in quantum mechanics, the various positions in space, $\mathbf{r}_{0}$, should be the eigenvalues of some position operator (observable), $\hat{\mathbf{r}}$. To pin down elements of the problem, we first consider a massive relativistic particle of zero spin. For such a particle the Lorentzinvariant scalar product is given by ${ }^{15}$

$$
\langle\psi \mid \phi\rangle=\int_{-\infty}^{\infty} \psi^{*}(\mathbf{p}) \phi(\mathbf{p}) \frac{d^{3} p}{\left[p^{2}+\left(M c_{0}\right)^{2}\right]^{1 / 2}}
$$

in the momentum representation. In the plasmariton case the particle mass (M) is given by Eq. (32). Within the framework of the relativistic scalar product the Hermitian operator

$$
\hat{\mathbf{r}}=i \hbar\left\{\nabla_{\mathbf{p}}-\frac{\mathbf{p}}{2\left[p^{2}+\left(M c_{0}\right)^{2}\right]}\right\}
$$

turns out to be an acceptable position operator for a Klein-Gordon particle. In the non-relativistic (NR) limit one regains the naive position operator $\hat{\mathbf{r}}_{N R}=i \hbar \nabla_{\mathbf{p}}$. The operator in Eq. (53) satisfies the basic dyadic commutator relation

$$
[\hat{\mathbf{r}}, \hat{\mathbf{p}}]=i \hbar \hat{\mathbf{1}}
$$

where $\hat{\mathbf{1}}$ is the unit matrix operator. In the momentum representation, where the eigenvalue equation reads

$$
\hat{\mathbf{r}}(\mathbf{p}) \psi_{\mathbf{r}_{0}}(\mathbf{p})=\mathbf{r}_{0} \psi_{\mathbf{r}_{0}}(\mathbf{p}),
$$

the (unnormalizable) eigenfunction belonging to the eigenvalue $\mathbf{r}_{0}$ is given by

$$
\psi_{\mathbf{r}_{0}}(\mathbf{p})=\left[p^{2}+\left(M c_{0}\right)^{2}\right]^{1 / 4} \exp \left(-\frac{i}{\hbar} \mathbf{r}_{0} \cdot \mathbf{p}\right),
$$

apart from an arbitrary multiplicative factor. In configuration space the eigenfunction takes the form

$$
\psi_{\mathbf{r}_{0}}(\mathbf{r})=2(2 \pi \hbar)^{-2} \frac{\left(M c_{0}\right)^{3 / 2}}{\left|\mathbf{r}-\mathbf{r}_{0}\right|} F\left(Q_{c}\left|\mathbf{r}-\mathbf{r}_{0}\right|\right),
$$

where

$$
F\left(Q_{c}\left|\mathbf{r}-\mathbf{r}_{0}\right|\right)=\int_{0}^{\infty} x\left(1+x^{2}\right)^{1 / 4} \sin \left(Q_{c}\left|\mathbf{r}-\mathbf{r}_{0}\right| x\right) d x .
$$

It appears that the function $\mathrm{F}$ only depends on the ratio between $\left|\mathbf{r}-\mathbf{r}_{0}\right|$ and the Compton wavelength of the plasmariton, $\lambda_{c}=h /\left(M c_{0}\right)$. Since $F(z) \approx z^{-5 / 4} \exp (-z)$ for $z>>1$, the wave function of a scalar particle 
definitely localized at $\mathbf{r}_{0}$ is smeared out in configuration space over a region of linear extension as the Compton wavelength around the point of localization. In the massless limit $(M \rightarrow 0)$, the wave function exhibits a power-law dependence $\left|\mathbf{r}-\mathbf{r}_{0}\right|^{-7 / 2}$ around the point of localization.

So far, we have ignored the vector character of the problem. For a transverse plasmariton we may try a three-vector momentum space wave function of the type

$$
\psi_{\mathbf{r}_{0}}(\mathbf{p} ; s)=\left[p^{2}+\left(M c_{0}\right)^{2}\right]^{1 / 4} \mathbf{e}_{\mathbf{p} s} \exp \left(-\frac{i}{\hbar} \mathbf{r}_{0} \cdot \mathbf{p}\right),
$$

where $\mathbf{e}_{\mathbf{p} s}(s=1$ or 2$)$ is one of two transverse unit vectors. The longitudinal unit vector, which points in the $\mathbf{p}$-direction, is denoted by $\mathbf{e}_{\mathbf{p} 3}$ below. When the gradient operator, $\nabla_{\mathbf{p}}$, acts on the wave function, it mixes in a longitudinal component, and this effect in itself makes it problematic (impossible) to take the $\hat{\mathbf{r}}$-operator in Eq. (53) as the plasmariton position operator. Instead, it is perhaps natural to use the dyadic quantity

$$
\hat{\mathbf{r}}_{i j}=i \hbar\left\{\delta_{i j}\left(\nabla_{\mathbf{p}}-\frac{\mathbf{p}}{2\left[p^{2}+\left(M c_{0}\right)^{2}\right]}\right)-\sum_{s=1}^{3}\left[\nabla_{\mathbf{p}}\left(\mathbf{e}_{\mathbf{p} s}\right)_{i}\right]\left(\mathbf{e}_{\mathbf{p} s}\right)_{j}\right\}
$$

as a candidate for a plasmariton position operator. ${ }^{7}$ This choice leads to problems in configuration space, however, because the related wave function (Fourier integral transform of Eq. (59)) is not convergent. If one tries to use the standard regularization procedure the divergence-free demand on the wave function cannot be maintained.

The photon position operator problem cannot be detached from the spatial localization issue of photons emitted from a source (microscopic or mesoscopic). In the field-quantized description the relation between the transverse electric field operator, $\hat{\mathbf{E}}_{T}$, and the transverse current density operator, $\hat{\mathbf{J}}_{T}$, can be written as follows:

$$
\hat{\mathbf{E}}_{T}(\mathbf{r}, t)=\mu_{0} \int_{-\infty}^{\infty} g\left(\left|\mathbf{r}-\mathbf{r}^{\prime}\right|, t-t^{\prime}\right) \cdot \frac{\partial}{\partial t^{\prime}} \hat{\mathbf{J}}_{T}\left(\mathbf{r}^{\prime}, t^{\prime}\right) d^{3} r^{\prime} d t^{\prime}
$$

If one now replaces $\hat{\mathbf{J}}_{T}$ by $\hat{\mathbf{J}}$ with the help of the transverse delta function the "propagator" relation

$$
\hat{\mathbf{E}}_{T}(\mathbf{r}, t)=\mu_{0} \int_{-\infty}^{\infty} \overleftrightarrow{\mathbf{D}}_{T}\left(\mathbf{r}-\mathbf{r}^{\prime}, t-t^{\prime}\right) \cdot \frac{\partial}{\partial t^{\prime}} \hat{\mathbf{J}}\left(\mathbf{r}^{\prime}, t^{\prime}\right) d^{3} r^{\prime} d t^{\prime}
$$

emerges. Here, the transverse propagator has the explicit dyadic form ${ }^{8}$

$$
\overleftrightarrow{\mathbf{D}}_{T}(\mathbf{R}, \tau)=-\frac{1}{4 \pi R} \delta\left(\frac{R}{c_{0}}-\tau\right)\left(\overleftrightarrow{\mathbf{U}}-R^{-2} \mathbf{R R}\right)+\frac{c_{0}^{2} \tau}{4 \pi R^{3}} \Theta(\tau) \Theta\left(\frac{R}{c_{0}}-\tau\right)\left(\overleftrightarrow{\mathbf{U}}-3 R^{-2} \mathbf{R R}\right)
$$

where $\Theta$ is the Heaviside step function. The form in Eq. (63) tells us that the transverse photon localization (in general) is limited to the extension of the rim zone, and that one cannot maintain the Einstein causality when compressing the source domain artificially from $\hat{\mathbf{J}}_{T}$ to $\hat{\mathbf{J}}$. Eq. (63) has served as a starting point for microscopic photon tunneling analyses in near-field electrodynamics. ${ }^{16}$

\section{REFERENCES}

[1] Landau, L. and Peierls, R. Zeitschr. Phys. 62, 188 (1930).

[2] Oppenheimer, J. R. Phys. Rev. 38, 725 (1931).

[3] Keller, O. Progr. Opt. 50, 51 (2007).

[4] Bialynicki-Birula, I. Progr. Opt. 36, 245 (1996).

[5] Goldstein, H., [Classical Mechanics], Addison-Wesley, London (1980).

[6] Born, M. and Wolf, E., [Principles of Optics], Cambridge Univ., Cambridge (1999).

[7] Hawton, M. Phys. Rev. A59, 3223 (1999).

[8] Keller, O. Phys. Rep. 411, 1 (2005). 
[9] Keller, O. Phys. Rev. A76, 062110 (2007).

[10] Keller, O. Phys. Rev. A58, 3407 (1998).

[11] Keller, O. Phys. Rev. A62, 022111 (2000).

[12] Keller, O. Laser Part. Beams 26, 287 (2008).

[13] Cohen-Tannoudji, C., Dupont-Roc, J., and Grynberg, G., [Photons and Atoms. Introduction to Quantum Electrodynamics], Wiley, New York (1989).

[14] Mandl, F. and Shaw, G., [Quantum Field Theory], Wiley, Chichester (2001).

[15] Weinberg, S., [The Quantum Theory of Fields, Vol. I, Foundations], Cambridge Univ., Cambridge (1996).

[16] Keller, O. Phys. Rev. A60, 1652 (1999). 